\title{
Growth and Initial Experiments Demonstrating Watt Level Output From Yb:YAG Planar Waveguides Grown by Pulsed Laser Deposition
}

\author{
Stephen J. Beecher ${ }^{1}$, James A. Grant-Jacob ${ }^{1}$, Tina L. Parsonage ${ }^{1}$, Ping Hua ${ }^{1}$, Amol Choudhary ${ }^{2}$, \\ Jacob I. Mackenzie ${ }^{1}$, Dave P. Shepherd ${ }^{1}$, and Robert W. Eason ${ }^{1}$ \\ 1. Optoelectronics Research Centre, University of Southamtpon, Southampton, SO17 1BJ, UK \\ 2. CUDOS, School of Physics, University of Sydney, NSW, Australia, 2006

\section{S.J.Beecher@soton.ac.uk}

\begin{abstract}
We report on the growth and initial characterization of pulsed laser deposited Yb:YAG. When used as a planar waveguide laser slope efficiencies of at least $34 \%$ are observed for a $45 \%$ reflectivity output coupler.

OCIS codes: (310.0310) Thin films; (230.7390) Optical fabrication; (140.3480) Lasers, diode-pumped; (230.7390) Waveguides, planar; (130.0130) Integrated optics; (140.3380) Laser materials; (140.5680) Rare earth and transition metal solid-state lasers.
\end{abstract}

\section{Introduction}

Crystalline planar waveguides combine many of the properties of fiber and bulk laser systems, with confinement of the pump and laser modes leading to the potential for high slope efficiencies, low thresholds and simple thermal management. Their adoption has however been limited due to difficulties in fabrication, typically involving the precision polishing of large areas to the very high surface qualities required for direct bonding. Pulsed laser deposition (PLD) allows for the epitaxial growth of thin crystal layers on a substrate via the ablation of target material with a pulsed laser. Material composition can be changed, either in steps or continuously by the use of multiple targets each contributing to the growth. PLD has previously been used to grow YAG crystal films [1], but research on this crystal grown with this method appears to have been discontinued due to difficulties with material ablation and the low index contrast attainable, which limits the numerical aperture of the waveguide.

Here we present work on the growth of Yb:YAG on $\langle 100\rangle$-oriented YAG substrates, the spectroscopy of the films and initial laser performance.

\section{Fabrication}

To aid in the ablation of the source material we chose a pressed ceramic target. It was our belief that the scattering of the $\mathrm{KrF}$ excimer radiation incident on the target decreases the penetration depth of the laser reducing the ablation threshold for this high bandgap material with relatively low linear absorption at $248 \mathrm{~nm}$. In this instance the target was a 1.4 at.\% Yb-doped YAG with ablation carried out at $1.6 \mathrm{~J} / \mathrm{cm}^{2}$ at a repetition rate of $20 \mathrm{~Hz}$, pulse duration of $20 \mathrm{~ns}$ with an oxygen background of $2 \times 10^{-2} \mathrm{mBar}$. The substrate was heated from the backside by a $\mathrm{CO}_{2}$ laser to a temperature of $\sim 900{ }^{\circ} \mathrm{C}$. Growth was comparatively quick with the $15 \mu \mathrm{m}$ thick guide used in this work taking 4 hours to deposit. After growth, the sample had the facets polished plane parallel resulting in a waveguide length of $8 \mathrm{~mm}$.

\section{Material characterization}

X-ray diffraction (XRD) performed on the sample confirms a highly crystalline film, which follows the orientation of the substrate and a pole figure analysis further confirms the existence of predominantly the cubic phase following the $\langle 100>$-orientation of the substrate, see Fig. 1. A small shift in the (400) YAG peak on the XRD is thought to be the result of a slight Al deficiency in the film, which occurs as a result of imperfect stoichiometric transfer from the target to the substrate. The fluorescence from the film when pumped by a laser diode tuned to the blue side of the zero phonon line $(\sim 960 \mathrm{~nm})$ was captured by a multimode optical fiber and measured by an optical spectrum analyzer (Ando AQ6317) following the technique previously reported in [2]. The use of a thin (15 $\mu \mathrm{m})$ film almost completely removes the spectrally dependent contributions from reabsorption losses, which typically skew measurements of fluorescence from quasi-four-level materials towards the red side of the spectrum. The FüchtbauerLadenburg equation was applied to this assuming a text book fluorescence lifetime of $950 \mu$ s to convert this fluorescence measurement to an emission cross section. This results in a $1030 \mathrm{~nm}$ peak emission cross-section slightly lower than typically reported for bulk Yb:YAG (our measured peak is $1.7 \mathrm{pm}^{2}$ ) and slightly increased 
emission on the zero-phonon line at $969 \mathrm{~nm}$ (our measured peak is $0.8 \mathrm{pm}^{2}$ ). Two possibilities for this deviation exist, either the compositional difference is impacting the spectroscopy of the film or the majority of reported measurements have some skew in the data due reabsorption of some of the fluorescence. Fluorescence measurements conducted on a Yb:YAG waveguides fabricated from bulk polished YAG are underway to ascertain if the composition differences are solely responsible.
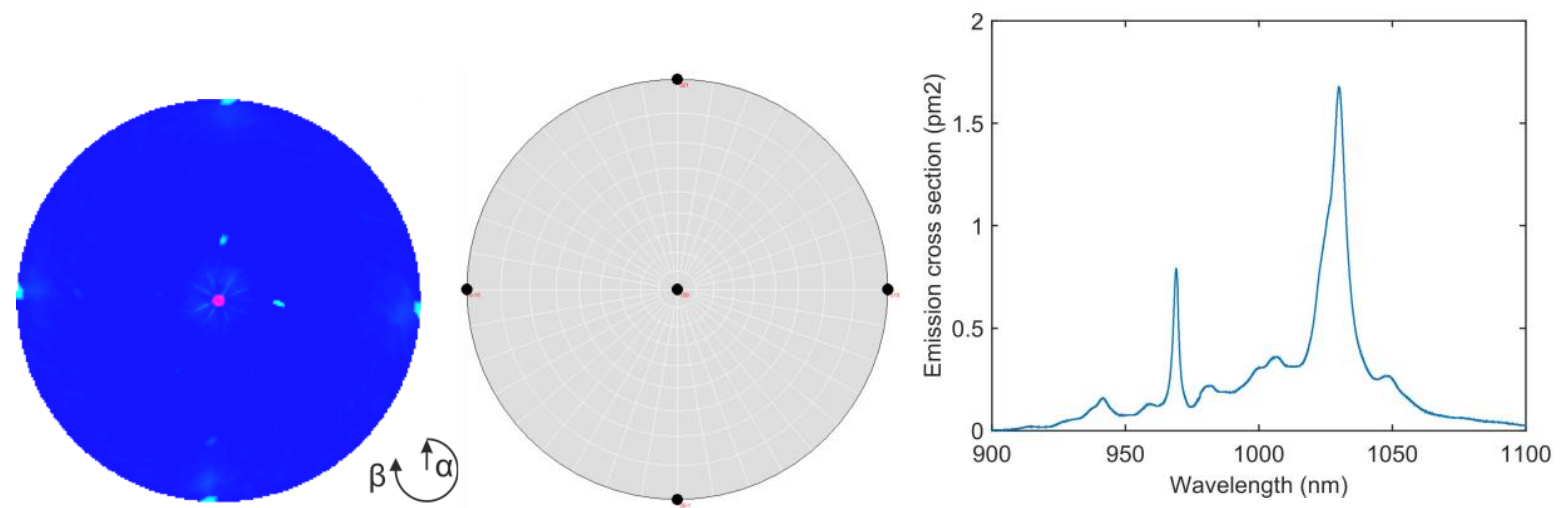

Fig 1. (Left) Pole figure of the Yb:YAG film. (Centre) A modelled pole figure for perfect <100>-YAG. (Right) the emission cross section calculated using the Füchtbauer-Ladenburg equation from the measured fluorescence spectra assuming a lifetime of $950 \mu \mathrm{s}$.

\section{Laser Performance}

The sample was mounted on a water-cooled heatsink and a pair of mirrors brought into close proximity to the polished waveguide facets. At the pump input side a thin mirror (HT 950-990 nm, HR 1030-1100 nm) was used to minimize aberration of the pump spot and on the other side two output couplers were trialed in addition to feedback being provided by the Fresnel reflection at the Yb:YAG-air interface. A nominally $976 \mathrm{~nm}$ diode bar was cooled to $10^{\circ} \mathrm{C}$ and driven at a low current such that the center wavelength of emission was $969 \mathrm{~nm}$ to allow for zero phonon line pumping. Due to the short waveguide length and comparatively low $\mathrm{Yb}$ concentration a significant amount of the pump light was transmitted directly through the guide without absorption. The guide is almost exactly one absorption length for the peak of the $969 \mathrm{~nm}$ absorption line and due to the diode emission spectrum being broader than this absorption feature the resulting pump absorption is less than half for a single pass. Cylindrical lenses were

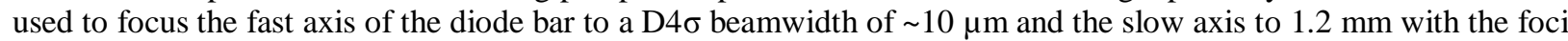
separated by $\sim 2 \mathrm{~mm}$ in air resulting in the slow axis focus being approximately in the center of the sample when the fast axis is optimally coupled into the waveguide. The output of the waveguide laser as a function of pump power for the different output couplers trialed is presented in Fig. 2. It should be noted that the absorbed power assumes perfect coupling of the diode bar into the waveguide, which due to the more than 100:1 aspect ratio of the pump beam is likely an over estimate and no account has been made for the reduction of ions in the ground state when a sufficient population inversion to reach threshold occurs. Note should be taken of the small difference in slope efficiency between $10 \%$ and 55\% output coupling implying a low propagation loss for the laser mode. Also of note is the spatial intensity distribution of the laser mode. No fine structure is observed in the unguided direction, indicating a highly uniform film with minimal defects. This is in contrast to some of our previous work in $\mathrm{Yb}_{2} \mathrm{Y}_{2} \mathrm{O}_{3}$ where porosity and strain at grain boundaries resulted in significant fine structure in the laser mode [2]. 

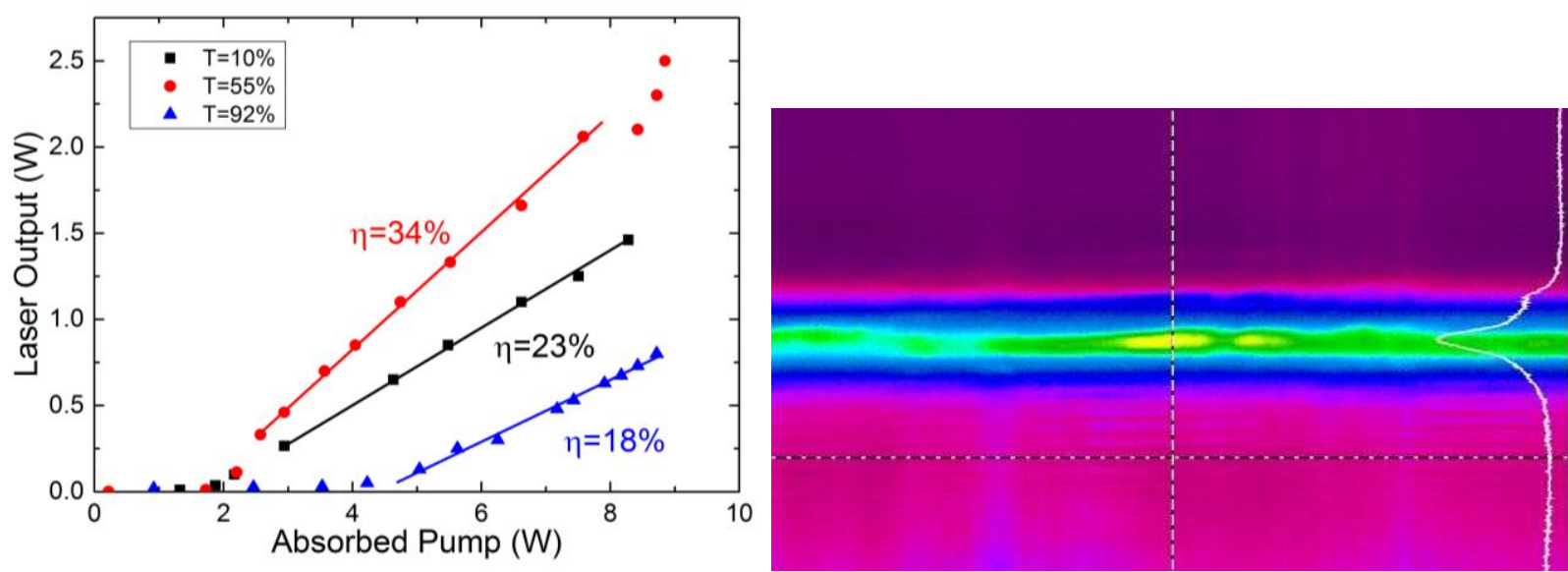

Fig 2. (Left) laser performance for different pump powers and output couplers. (Right) Image of the waveguide laser mode

\section{Conclusions}

We have demonstrated that high-quality Yb:YAG thin films can be grown by PLD at a very fast deposition rate, potentially making this technique suitable for volume production of planar waveguides and thin disks. The near perfect lattice matching between the undoped YAG substrate and the Yb-doped thin film results in minimal lattice defects or strain, and should allow for the growth of thicker films without issues relating to strain relief such as cracking etc. Work is underway to improve pump absorption by increasing the dopant concentration and to investigate the growth of thicker films for thin disc applications.

\section{References}

[1] H. Kumagai, K. Adachi, M. Ezaki, K. Toyoda and M. Obara, "Epitaxial growth of Nd:YAG thin films by pulsed laser deposition,” Appl Surf Sci 109-110, 528-532 (1997)

[2] S. J. Beecher, T. L. Parsonage, J. I. Mackenzie, K. A. Sloyan, J. A. Grant-Jacob, and R. W. Eason, "Diode-end-pumped 1.2 W Yb: $\mathrm{Y}_{2} \mathrm{O}_{3}$ planar waveguide laser," Opt. Express 22, 22056-22061 (2014) 